\title{
1 Bottom-up and top-down factors of motion direction learning transfer
}

2 Yang Zhang ${ }^{1}$, Yan-Fang Yuan ${ }^{1}$, Xun $\mathrm{He}^{2}$, and Gong-Liang Zhang ${ }^{1 *}$

$3 \quad{ }^{1}$ Department of Psychology, School of Education, Soochow University, Suzhou, Jiangsu,

4 China

$5 \quad{ }^{2}$ Department of Psychology, Faculty of Science and Technology, Bournemouth University,

6 Poole, Dorset, UK

$7 \quad *$ Email for correspondence: Glenn@suda.edu.cn

\section{Abstract}

9 Perceptual learning of motion discrimination has long been believed to be

10 motion direction specific. However, recent studies using a double-training paradigm,

11 in which the to-be-transferred condition was experienced through practicing an

12 irrelevant task, found that perceptual learning in various visual tasks, including

13 motion direction discrimination, can transfer completely to new conditions. This

14 transfer occurred when the transfer stimulus was subconsciously presented, or when

15 top-down attention was allocated to the transfer stimulus (which was absent). In the

16 current study, observers were exposed subconsciously, or directed top-down attention,

17 to the transfer motion direction, either simultaneously or successively with training.

18 Data showed that motion direction learning transferred to the transfer direction, and

19 suggest that motion direction learning specificity may result from under-activations of

20 untrained visual neurons due to insufficient bottom-up stimulation and/or lack of

21 top-down attention during training. These results shed new light on the neural 
1 mechanisms underlying motion perceptual learning and provide a constraint for

2 models of motion perceptual learning.

3 Keywords: perceptual learning; motion direction; specificity; learning transfer;

4 double training

\section{Introduction}

Visual perceptual learning refers to improvement in perceptual performance as

7 a result of training or experience. It takes place in various visual tasks involving basic

8 visual features, such as contrast, orientation, and motion information (see Fahle \&

9 Poggio, 2002; Gilbert, Sigman, \& Crist, 2001, for reviews). One hallmark of

10 perceptual learning is that it is typically specific to the trained locations and features

11 (e.g., orientation) (Ahissar \& Hochstein, 1997; Ball \& Sekuler, 1987; Fahle, 1994;

12 Karni \& Sagi, 1991). This is often taken as an evidence that V1 neurons are

13 responsible for a large degree of specificity (Fiorentini \& Berardi, 1981; Karni \& Sagi,

14 1991; Schoups, Vogels, \& Orban, 1995; Schoups, Vogels, Qian, \& Orban, 2001)

15 because neurons in V1 are most retinotopic (Tootell, Silverman, Switkes, \& De Valois,

16 1982) and orientation selective (Hubel \& Wiesel, 1959). This hypothesis has also been

17 supported by neurophysiological studies which found that training could sharpen

18 neuronal orientation tuning in the primary visual cortex (Schoups et al., 2001; Teich \&

19 Qian, 2003). However, Ghose, Yang, and Maunsell (2002) did not find any orientation 20 tuning changes in either V1 or V2 neurons. Petrov, Dosher, and Lu (2005) further 
1 suggested that modification of early cortical representations might not be necessary

2 for orientation discrimination learning. Instead, the read-out connections to a decision

3 unit were proposed to be reweighted through training. Following these pioneering

4 studies, during the past decade, many procedures have been developed to show that

5 learning can indeed transfer from trained to untrained conditions (see Herzog et al.,

6 2017, for a review), suggesting that perceptual learning may occur in higher stages of

7 visual processing than the sensory cortices. Among these procedures, the

8 “double-training" or "training-plus-exposure" procedures (Xiao et al., 2008; Zhang et

9 al., 2010) are two of the most widely used. With a "double-training" procedure, Xiao

10 et al. (2008) employed a contrast training procedure at one location, and

11 task-irrelevant location training at a different location. The results showed that the

12 additional location training enabled a complete learning transfer across locations. In

13 another study, Zhang et al. (2010) adopted a training-plus-exposure (TPE) procedure,

14 in which observers were trained at one orientation and passively exposed to a second

15 orientation. It was found that learning of orientation perception transferred completely

16 from the training orientation to the exposure orientation (i.e., the transfer orientation).

17 A rule-based learning model was proposed, stating that perceptual learning is most

18 likely a high-level rule-based learning process and thus potentially transferrable to

19 untrained conditions (Zhang et al., 2010). By default, the learned rules do not apply to

20 the untrained conditions automatically because the functional connectivity between

21 the low-level neurons (responding to the untrained conditions) and the high-level 
1 areas is usually unestablished, leaving the learning specific to the trained conditions.

2 However, if the untrained locations and features have been activated (e.g., via a

3 double training or TPE procedure), the learning rules will be applied to these

4 untrained locations and features due to the newly established functional connectivity

5 between the low visual cortices and the high-level areas, leading to a transfer of

6 learning to the untrained conditions (Xiao et al., 2008; Zhang et al., 2010).

$7 \quad$ Furthermore, in a more recent double-training study, Xiong, Zhang, \& Yu et al.

8 (2016) isolated the influence of bottom-up stimulation and top-down attention on

9 learning transfer with a continuous flash suppression (CFS) method (Tsuchiya \&

10 Koch, 2005). Participants were trained on a task (e.g., Vernier discrimination) at one

11 location, and were exposed to the same stimulus sub-consciously at another location

12 (the bottom-up stimulation condition), or were asked to attend to another location

13 where no stimulus was presented (the top-down condition). It was shown that both the

14 bottom-up stimulation and top-down attention manipulations independently enabled

15 significant learning transfer, supporting the rule-based learning model (e.g., Zhang et

16 al., 2010).

Although Xiong, Zhang et al. (2016) questioned learning specificity that had

18 dominated perceptual learning research for decades, and offered new insight into the

19 rule-based learning models, some critical questions were not answered. First of all,

20 the Vernier discrimination and orientation discrimination tasks are arguably a type of

21 visual form processing. It remains unknown whether bottom-up stimulation and 
1 top-down attention could also facilitate transfer of motion perceptual learning.

2 Secondly, previous studies have demonstrated that perceptual skills might consolidate

3 off-line during overnight sleep (see Peigneux \& Smith, 2010, for a review). Thus the

4 functional connection mentioned in Xiong, Zhang et al.'s study (2016) may

5 established before consolidation because training and the bottom-up

6 stimulation/top-down attention manipulations were administered across blocks on the

7 same day. It is therefore unclear whether functional connection can also be established

8 after learning have already been consolidated.

9 To address the concerns mentioned above, the current study adopted the

10 paradigm used by Xiong, Zhang et al. (2016) to investigate the effect of bottom-up

11 stimulation and top-down attention on motion discrimination learning transfer. In the

12 bottom-up stimulation (of the untrained motion direction) condition, motion

13 discrimination was trained in one direction, with stimuli in the transfer direction being

14 rendered invisible with CFS (Tsuchiya \& Koch, 2005). In the top-down attention (to

15 the untrained motion direction) condition, participants were asked to voluntarily

16 attend to the transfer direction while there was, in fact, no stimulus presented. To

17 address the second issue, bottom-up stimulation and top-down attention were

18 separately applied either at the same time of training (the training and the

19 bottom-up/top-down manipulations in alternative blocks in the same session) or after

20 training (the bottom-up/top-down manipulations applied after five sessions of

21 training). If the same learning rule as found in previous studies (e.g., Xiong, Zhang, et 
1 al., 2016) also governs motion learning, we would expect a significant transfer of

2 motion learning in these two conditions mentioned above. Furthermore, if

3 transfer-facilitation effect of the bottom-up stimulation and top-down attention could

4 still be effective after learning has already been consolidated, the learning transfer

5 should be observed to be at the same magnitude no matter whether the manipulation

$6 \quad$ was applied simultaneously or sequentially.

\section{$7 \quad$ Materials and method}

8 Observers and apparatus

Thirty-one naïve volunteers from Peking University (aged 21.7 \pm 2.4 years; 16

10 male and 15 female) and eighteen from Soochow University (aged $22.2 \pm 2.0$ years; 6

11 male and 12 female) participated in the current study. There were at least six

12 observers in each experiment (see Table 1 for details). Five out of the six observers in

13 each of Experiments 1, 2A, 2B, 2C, and 3B were recruited from Peking University;

14 the remaining observers in these experiments were recruited from Soochow

15 University. All 6 observers in Experiment 3A were recruited from Peking University,

16 and all observers in Experiments 4 and 5 were recruited from Soochow University. All

17 observers had normal or corrected-to-normal vision (measured with a Tumbling E

18 chart), gave written informed consent. The study was approved by the academic and

19 ethics committees of Peking University and Soochow University, in agreement with 20 the Declaration of Helsinki. 
2 equipped with an NVIDIA Quadro FX 4600 graphics card were used. Stimuli were

3 presented on 21-inch Dell P1230 CRT monitors $(1600 \times 1200$ resolution, $0.25 \times 0.25$

$4 \mathrm{~mm}$ per pixel, $85-\mathrm{Hz}$ refresh rate). The mean luminance (average of minimal and

5 maximum luminance, measured with a ColorCal MKII photometer, Cambridge

6 Research Systems Ltd., Cambridge, UK) of the monitor was $41.5 \mathrm{~cd} / \mathrm{m}^{2}$ at Peking

7 University and $41.1 \mathrm{~cd} / \mathrm{m}^{2}$ at Soochow University (for more details about the monitors,

8 see Zhang et al., 2018). The luminance was linearized by an 8-bit look-up table.

9 Stimuli were prepared with MATLAB (MathWorks, Inc., Natick, MA, USA) and

10 Psychtoolbox-3 (Brainard, 1997; Pelli, 1997). Experiments were run in dimly lit

11 rooms, with a chin-and-head rest stabilizing the heads of the observers.

13 Experiments

14 Five experiments were conducted in this study and are summarized in Table 1.

15 All experiments measured the participants' motion direction discrimination thresholds

16 before (Session 1) and after training (Session 7 and Session 13), with five training

17 sessions between the measurements of thresholds (Sessions 2-6 and Sessions 8-12).

18 The training sessions took place across several days, with each training session

19 completed within a single day, and the next training session coming up one or two

20 days afterward. Before each experiment, there was a block of practice trials with a

21 staircase procedure (about 50 trials), showing motion stimuli in both the to-be-trained 
1 direction and the transfer direction. Experiment 1 measured the motion direction

2 learning specificity and recorded the baseline training performance. Experiment $2 \mathrm{~A}$

3 measured the effect of the bottom-up stimulation on learning transfer. Experiment 2B

4 was designed to exclude the alternative account that the bottom-up stimulation alone

5 can induce motion learning in the transfer direction. A further Experiment $2 \mathrm{C}$ was

6 included to exclude the possibility that transfer can be caused by noise stimulation

7 instead of the bottom-up stimulation in the transfer motion direction. 
Table 1. Experiments and tasks in the current study. In each experiment, motion direction discrimination thresholds were measured pre-training and post-training, with five training sessions in between. Various training designs were used across experiments. In Experiments 1-3B, there was only one training phase (Phase I), where the bottom-up/top-down manipulations were applied simultaneously with the main training when applicable. In the last two experiments, the bottom-up/top-down manipulations were applied subsequently in Phase II.

\begin{tabular}{|c|c|c|c|c|c|c|}
\hline & \multirow[b]{2}{*}{$\begin{array}{c}\text { Sample } \\
\text { size }\end{array}$} & \multicolumn{3}{|c|}{ Phase I } & \multicolumn{2}{|l|}{ Phase II } \\
\hline & & Session 1 & Sessions 2-6 (training) & Session 7 & Sessions 8-12 (training) & Session 13 \\
\hline Exp 1: Training baseline & 6 & Threshold & Motion discrimination & Threshold & & \\
\hline Exp 2A: Simultaneous bottom-up & 6 & Threshold & Motion discrimination \& color discrimination & Threshold & & \\
\hline Exp 2B: Bottom-up only & 6 & Threshold & Color discrimination & Threshold & & \\
\hline Exp 2C: Simultaneous noise & 6 & Threshold & Motion discrimination \& color discrimination & Threshold & & \\
\hline Exp 3A: Simultaneous top-down & 6 & Threshold & Motion discrimination \& motion detection & Threshold & & \\
\hline Exp 3B: Top-down only & 6 & Threshold & Motion detection & Threshold & & \\
\hline Exp 4: Subsequent bottom-up & 6 & Threshold & Motion discrimination & Threshold & Color discrimination & Threshold \\
\hline Exp 5: Subsequent top-down & 7 & Threshold & Motion discrimination & Threshold & Motion detection & Threshold \\
\hline
\end{tabular}


2 measured the effect of top-down attention on learning transfer. Experiment 3B was designed

3 to exclude the possibility that top-down attention alone can induce motion learning in the

4 transfer direction. Experiments 4 and 5 were utilized to explore whether the

5 transfer-facilitation effect of the bottom-up stimulation and top-down attention could still be

6 effective after learning have already been consolidated. To achieve this purpose,

7 Experiments 4 and 5 were carried out in two phases: motion direction training was

8 completed in Phase I; the bottom-up stimulation or top-down attention manipulation was

9 subsequently applied in Phase II, which was separated from Phase I by 1-2 days. In these

10 last two experiments, the post-training thresholds were measured twice in Sessions 7 and 13.

11 In all the experiments, for both the training and transfer motion directions, there were

12 at least 5 blocks of stimuli in Sessions 1, 7, and 13. If the variation was too large (i.e., the

13 standard error of the mean threshold values across blocks was larger than $10 \%$ of the

14 averaged threshold), we would consider the learning still unstable, and another block of

15 trials would be added immediately for both the trained and transfer motion directions at the

16 end of the session. Thus, each threshold in Sessions 1, 7, and 13 was averaged on the basis

17 of 5-6 staircases. The arithmetic mean across measurements for each condition was used as

18 the discrimination threshold (both pre-training and post-training). In Sessions 2-6 and

19 Sessions 8-12, each task listed in Table 1 was repeated for 10 blocks. For motion

20 discrimination, there were around 50 trials (with a staircase procedure) in each block. For

21 color discrimination and motion detection, each block had 50 trials. In Sessions 2-6 of 
1 Experiments 2A, 2C, and 3A, the two different tasks were switched every five blocks, with

2 the motion discrimination task always being conducted first.

3

4

\section{Stimuli}

Different stimuli were independently presented in the central vision of two eyes using a stereoscope, with motion stimuli in the non-dominant eye and white-noise patterns in the dominant eye. The motion stimulus consisted of 356 random white dots $\left(0.05^{\circ}\right.$ in diameter, about $83 \mathrm{~cd} / \mathrm{m}^{2}$ ) in an imaginative circular window with a diameter of $6^{\circ}$ over a gray (about $41 \mathrm{~cd} / \mathrm{m}^{2}$ ) background at the center of the screen. All dots moved in the same direction $\left(36^{\circ}\right.$ or $126^{\circ}$ in polar coordinates) at a speed of $6^{\circ}$ s. Each dot had a lifetime of 100 ms minus a random starting delay of 0-100 ms. When a dot reached its lifetime, a new dot was generated at a random position within the stimulus window following the same lifetime rule. When a dot traveled out of the stimulus window, the dot would disappear, and a new dot would enter from the other side of the window at a random position, again following the same lifetime rule.

To separate the potential bottom-up and top-down contributions, the CFS technique (Tsuchiya \& Koch, 2005) was applied in the training phases. The CFS configuration consisted of a square flashing white-noise pattern $\left(8.70^{\circ} \times 8.70^{\circ}\right)$ in the central vision of the dominant eye. The white-noise pattern, refreshed at $9.4 \mathrm{~Hz}$ for $953 \mathrm{~ms}$, consisted of randomly generated ovals of random sizes (the minor axis of the smallest ellipse was $0.08^{\circ}$, and the major axis of the largest ellipse was $0.33^{\circ}$ ) and random luminance (from 0 to about 
$183 \mathrm{~cd} / \mathrm{m}^{2}$ ). In the bottom-up stimulation condition, the motion stimuli, which moved

2 orthogonally to the trained motion direction, was presented in the non-dominant eye in half

3 of the trials (Figure 1). As the signal of the motion stimuli was much weaker than the

4 flashing noise patterns, the observers should not be able to detect the motion stimuli. To

5 confirm this suppression effect of CFS, eight observers (3 from Experiment 2A, 2 from

6 Experiment 2B, and 3 from Experiment 4) were tested with a two-alternative forced choice

7 (2AFC) motion detection task in which either a motion stimulus at the transfer direction or a

8 blank screen was presented to the non-dominant eye, with the flashing noise pattern

9 presented to the dominant eye ( 60 trials per block for 3 blocks). The observers were asked to

10 determine whether there was a motion stimulus. The result showed chance-level

11 performance $(0.49 \pm 0.01)$, suggesting a full suppression effect of the CFS used in the

12 current study. Therefore, we concluded that the results of learning transfer in Experiments

$132 \mathrm{~A}$ and 4 were not contaminated by the leakage of the motion perception. Furthermore, to

14 prevent the observers from perceiving the abrupt presentation of the motion stimuli, the

15 luminance of the motion stimuli increased gradually from the mean luminance (about 41

$\left.16 \mathrm{~cd} / \mathrm{m}^{2}\right)$ to the maximal luminance $\left(57.9 \mathrm{~cd} / \mathrm{m}^{2}\right)$ and then back to the mean luminance

17 following a Gaussian function in time. In the top-down attention condition, only a white

18 fixation was displayed in the non-dominant eye. 
a

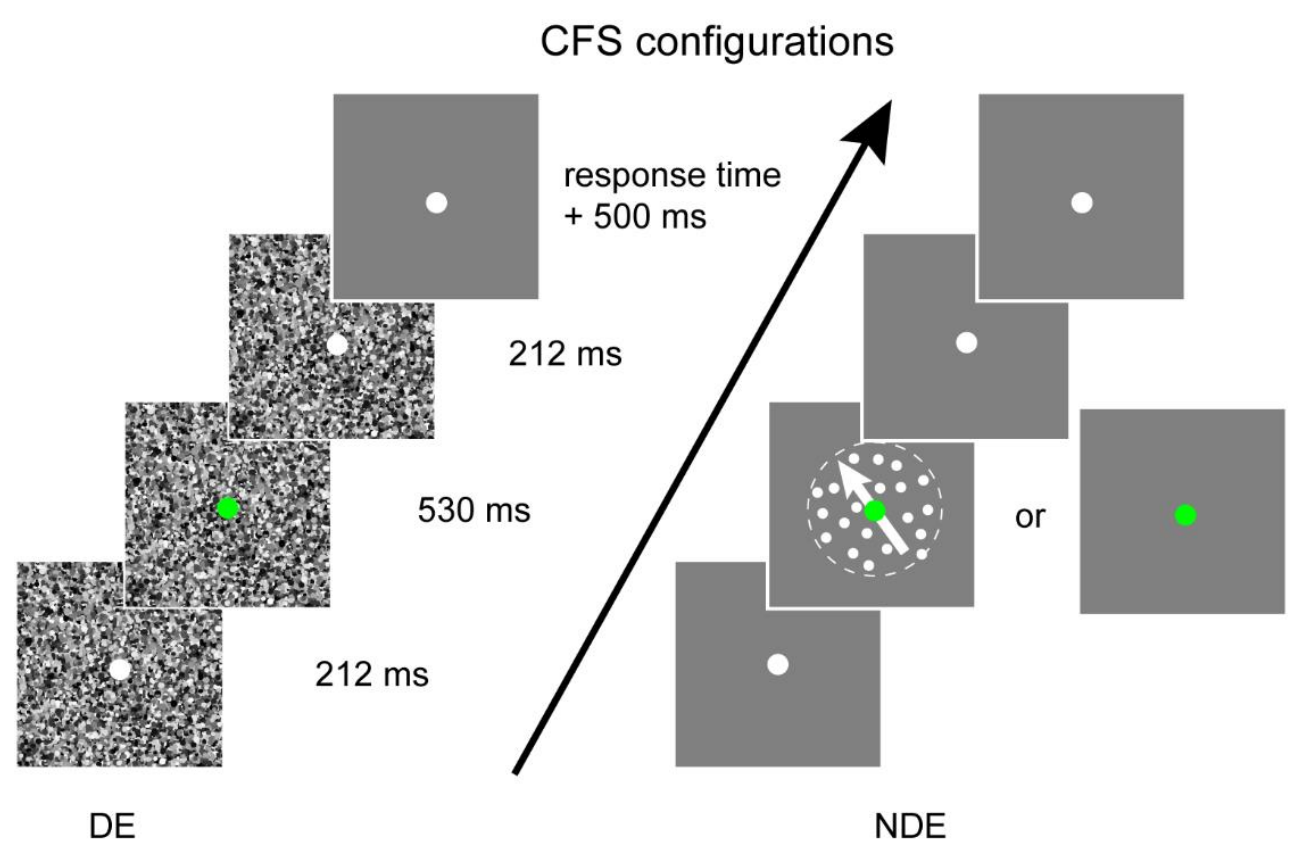

b

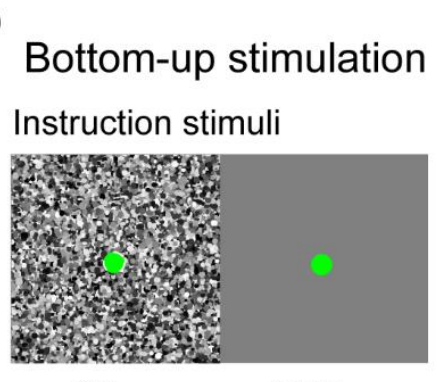

C Noise stimulation

d

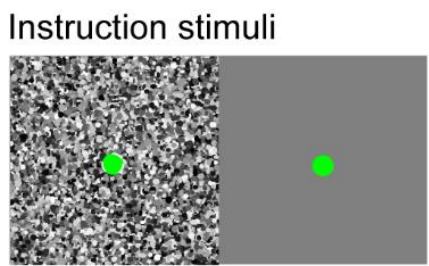

DE

NDE

Actual stimuli

Actual stimuli

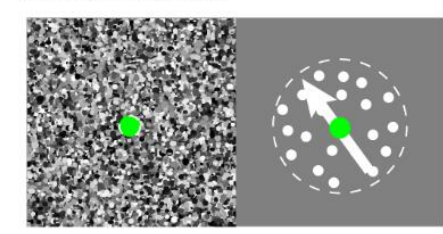

DE

NDE

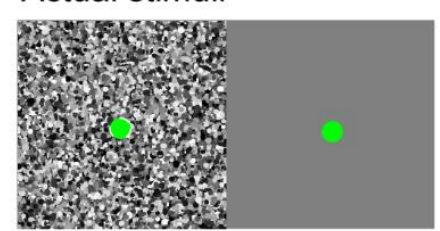

DE

NDE
Top-down attention

Instruction stimuli

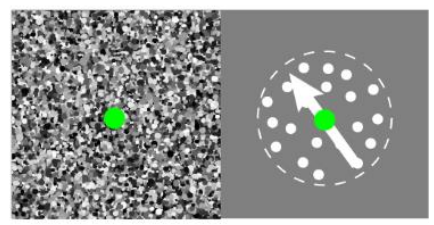

DE

NDE

Actual stimuli

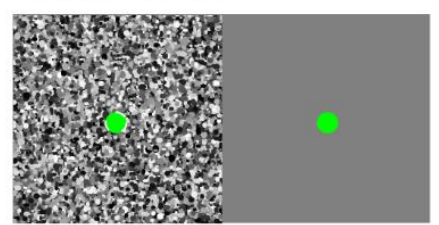

DE

NDE

2 Figure 1. Stimuli used in the current study. (a) Schematic diagram of continuous flashing

3 suppression (CFS) configurations, which were used in the bottom-up stimulation (actual

4 stimuli) and also in the top-down attention instruction. The flashing white-noise pattern was

5 presented for $953 \mathrm{~ms}$ in the dominant eye. At $213 \mathrm{~ms}$ after pattern onset, a motion stimulus

6 in the transfer motion direction was presented in the non-dominant eye for $530 \mathrm{~ms}$ in half of

7 the trials and was absent in the other half of the trials. The fixations in both eyes turned 
1 green or red at the same time when the motion stimulus (or a blank screen) was presented.

2 (b) Stimuli for the bottom-up stimulation manipulation. Bottom-up stimulation was achieved

3 subconsciously with the bottom-up stimuli presented in the non-dominant eye but not

4 mentioned in the instructions. (c) Stimuli in the noise stimulation condition. The motion

5 stimuli were not presented or mentioned in the instructions. (d) Stimuli for the top-down

6 attention manipulation. The top-down attention was achieved by requesting the participants

7 to discriminate the motion direction in the non-dominant eye (instruction stimuli), despite that

8 the motion stimulus was in fact not presented in the test (actual stimuli). DE: dominant eye;

9 NDE: non-dominant eye.

11 Tasks and procedures

12 Three tasks were used in the current study: a motion direction discrimination task, a

13 color discrimination task, and a motion detection task. In the motion direction discrimination

14 task, motion direction discrimination thresholds were measured with a temporal 2AFC,

15 3-down-1-up staircase procedure (with a convergence level of 79.4\%). In each trial, the

16 reference and test stimuli (reference direction $\pm \Delta$ direction) were sequentially presented for

$17506 \mathrm{~ms}$ in a random order with a 506-ms inter-stimulus interval in between. A small white

18 fixation point $\left(0.25^{\circ}\right.$ in diameter $)$ preceded each trial by $506 \mathrm{~ms}$ and remained on the screen

19 throughout the trial. The observers were asked to determine which of the two stimuli

20 contained a motion more clockwise in its direction. A brief auditory feedback was given on

21 incorrect responses. Each staircase consisted of four preliminary reversals and six 
1 experimental reversals (approximately 50 trials in total) and started with an initial motion

2 direction difference of $11.2^{\circ}$. This value was about twice the average threshold revealed in

3 previous studies using identical stimuli (Xiong, Xie, \& Yu, 2016), thus easily discriminable.

4 The step size of the staircase was 0.05 log-units. The geometric mean of the six

5 experimental reversals was taken as the threshold for each staircase.

The color discrimination task was used to examine the effect of the bottom-up

7 stimulation and noise stimulation manipulations on the motion direction learning transfer. In

8 both of the bottom-up stimulation and noise stimulation conditions, the color of the fixation

9 dot (in both eyes) randomly changed to green or red for $530 \mathrm{~ms}$ from $212 \mathrm{~ms}$ after the onset

10 of the 953-ms CFS noise pattern (see Figure 1a). The observers were asked to discriminate

11 the color of this fixation color change (instruction stimuli shown in Figure 1b). No feedback

12 was provided. In the bottom-up stimulation condition, the stimulus in the non-dominant eye

13 was either only a fixation dot or a motion stimulus moving orthogonally to the trained

14 motion directions (also with a fixation dot; Figure 1b). In the noise stimulation condition,

15 the motion stimulus was never displayed (Figure 1c).

16 After having completed the testing, eighteen of the observers who participated in the

17 bottom-up stimulation condition were asked whether they saw a motion stimulus while

18 performing the color discrimination task. Only two observers reported having noticed a

19 motion stimulus occasionally but reported wrong motion directions, showing very limited

20 awareness of the motion stimuli. Because the observers did not notice the existence of the 
1 motion stimuli, the motion stimuli stimulated the visual cortex at the transfer motion

2 direction without being consciously perceived.

The motion detection task was used to engage top-down attention to the transfer

4 motion direction and check its effect on learning transfer. In this task, the color of the

5 fixation dot was always white, with a blank screen always displayed to the non-dominant

6 eye (actual stimuli in Figure 1d). To convince the observers that the motion stimuli were

7 present in half of the trials, in the first practice trial, a motion stimulus in the direction

8 orthogonal to the training direction was shown in the non-dominant eye with maximal

9 luminance (instruction stimuli in Figure 1d). The luminance decreased over trials until

10 observers reported that they could not detect the motion stimulus anymore. The observers

11 were told that the nearly undetectable stimulus towards the end of the practice would be

12 used in the experiment. The observers were then asked to report, or to guess if they had to,

13 whether a motion stimulus was shown by making a key press. Fake feedback with a random

14 accuracy score between $55 \%$ and $70 \%$ was provided by the end of each block. This

15 paradigm ensured the top-down attention being directed to the transfer motion direction

16 without any bottom-up stimulation.

\section{Results}

Experiment 1: Baseline performance - Motion direction learning specificity

19 Six subjects took part in this experiment, and training significantly reduced the threshold by $42.5 \pm 4.5 \%$ (mean $\pm \mathrm{SE}$, mean percent improvement $=[$ threshold pre-training -

21 threshold post-training $] /$ threshold pre-training $)$ in the trained motion direction $\left(t_{5}=9.37, p<.001\right.$, 
$195 \% \mathrm{CI}=30.8 \%$ to $54.1 \%$, one-sample $t$-tests against 0 in in this and later analyses if not

2 specified; Cohen's $d=3.82$; Figure 2). It is worth noting that $t$-test may not be an

3 appropriate method for ratio data analysis because ratio data may not be normally distributed.

4 Therefore, to provide additional converging evidence, a Wilcoxon signed-rank test was also

5 performed, and a consistent statistical result was found $(p=.028)$. Training reduced the

6 motion direction discrimination threshold in the (untrained) orthogonal direction only by

$7 \quad 14.0 \pm 3.3 \%\left(t_{5}=4.19, p=.009,95 \% \mathrm{CI}=5.4 \%\right.$ to $22.5 \% ; p=.028$ with a Wilcoxon

8 signed-rank test; Cohen's $d=1.71$ ). The improvement was significantly weaker in the

9 transfer motion direction than in the trained motion direction $\left(t_{5}=11.62, p<.001,95 \% \mathrm{CI}=\right.$

$1022.2 \%$ to $34.8 \% ; p=.028$ with a Wilcoxon signed-rank test; Cohen's $d=4.75$ ), indicating

11 that the learning only transferred partially from the trained to the transfer direction. This

12 shows the motion-direction learning specificity.

13 A transfer index (TI) was calculated to compare the transfer effects between motion

14 directions. TI was defined as the improvement at the transfer condition divided by the

15 improvement at the trained condition, with $\mathrm{TI}=0$ indicating a complete learning specificity

16 (no transfer at all), and TI = 1 indicating a complete learning transfer. If a TI value was more

17 than 3 SDs away from the group average, it was considered an outlier and was replaced by

18 the theoretical maximum or minimum, which was 1 or 0 respectively. The usage of the

19 minimum or the maximum depended on whether the TI value was more than 3 SDs above or

20 below the group average. According to this criterion, only one participant in Experiment 4

21 exhibited an outlier TI value $(\mathrm{TI}=2.83)$. In the baseline condition, $\mathrm{TI}=0.31 \pm 0.06$, which 
1 was significantly larger than $0\left(t_{5}=5.43, p=.003,95 \% \mathrm{CI}=.16\right.$ to $.45 ; p=.028$ with a

2 Wilcoxon test; Cohen's $d=2.22$ ). This significant performance improvement in the

3 untrained orthogonal direction may be due to some unspecific factors irrelevant to the

4 motion perception (e.g., procedure learning).

a

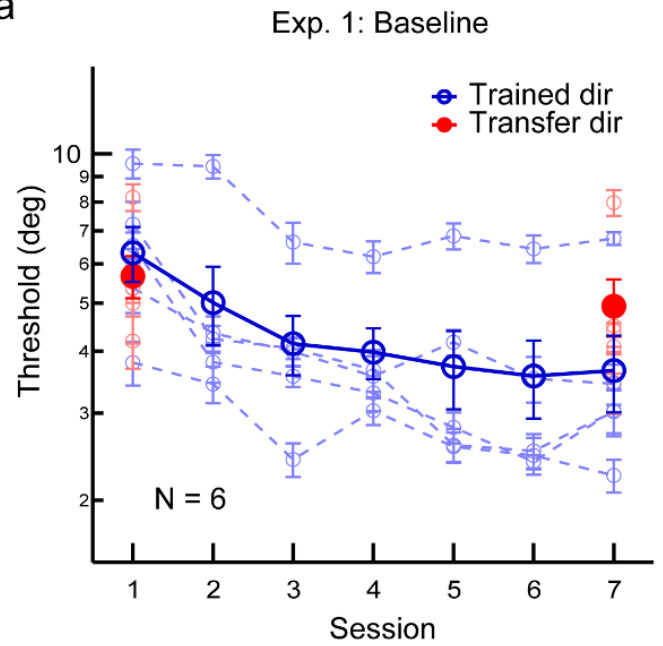

b

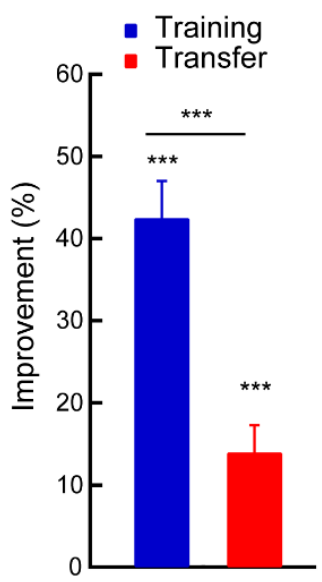

Exp. 1

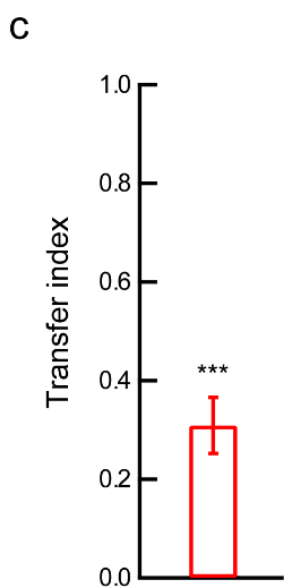

Exp.1

Figure 2. Results of Experiment 1, showing the baseline performance of the motion direction learning. (a) The mean and individual learning and transfer data. Individual data are plotted (dash lines) together with the averaged results (solid line). (b) Performance improvements in the trained and transfer motion directions. (c) Transfer index in the baseline condition. Error bars indicate \pm 1 standard errors of the means. ${ }^{* *} p<.001$.

\section{Experiment 2: Learning transfer - Effect of bottom-up stimulation}

Zhang and Yang (2014) demonstrated that motion discrimination learning could completely transfer to an untrained direction orthogonal to the trained direction if the observers received exposure to the transfer motion direction in a motion-irrelevant secondary task. In the current study, the observers received the motion direction training and 
1 the bottom-up stimulation in the transfer motion direction in separate blocks of the same

2 sessions (Experiment 2A). The results are summarized in Figure 3. Five sessions of training

3 reduced the motion discrimination threshold in the trained motion direction significantly by

$439.6 \pm 3.7 \%\left(t_{5}=10.63, p<.001,95 \% \mathrm{CI}=30.1 \%\right.$ to $49.2 \% ; p=.028$ with a Wilcoxon test;

5 Cohen's $d=4.34$ ), as well as in the untrained but bottom-up stimulated orthogonal motion

6 direction by $32.5 \pm 2.4 \%\left(t_{5}=13.30, p<.001,95 \% \mathrm{CI}=26.2 \%\right.$ to $38.8 \% ; p=.028$ with a

7 Wilcoxon test; Cohen's $d=5.43$ ) (Figures 3a and 3d). Transfer was substantial but not

8 complete, as suggested by a significant difference between the improvements in the two

9 directions $\left(t_{5}=4.77, p=.005,95 \% \mathrm{CI}=3.3 \%\right.$ to $11.0 \% ; p=.028$ with a Wilcoxon test;

10 Cohen's $d=1.95$ ), leading to a TI of $0.83 \pm 0.03$ (Figure 3e).

a

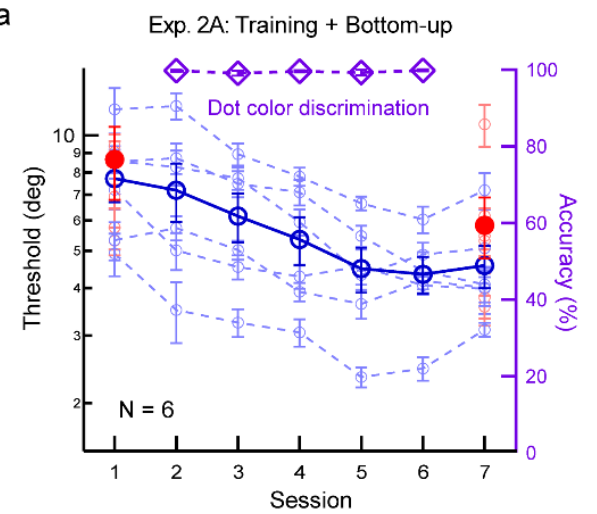

C

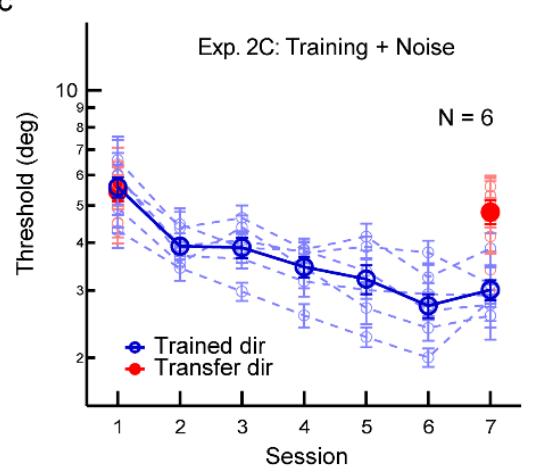

b

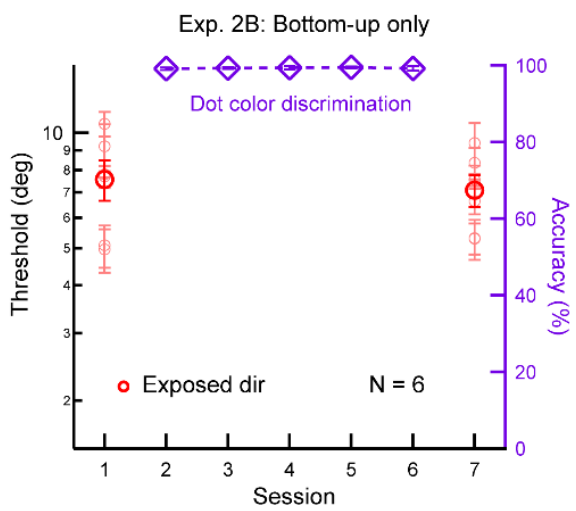

d

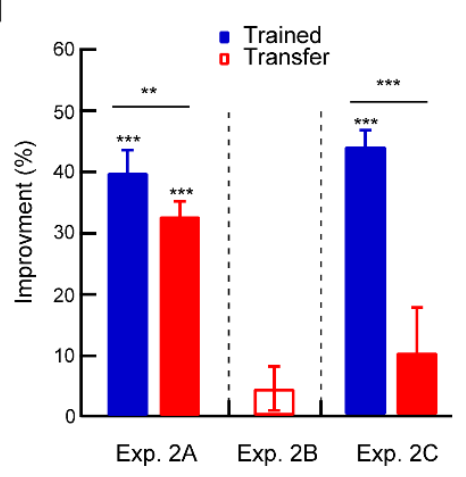

e

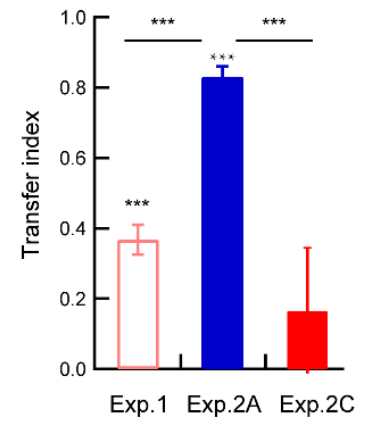


1 Figure 3. Results of Experiment 2, illustrating the performance during motion direction

2 learning and the effect of the simultaneous bottom-up stimulation in the transfer motion

3 direction. (a) The mean (solid line) and individual (dash lines) learning and transfer data with

4 the bottom-up stimulation in the transfer motion direction (Experiment 2A). (b) Results of the

5 control experiment (Experiment 2B), which applied the bottom-up stimulation but not the

6 motion direction training. (c) Results of another control experiment (Experiment 2C), which

7 employed the motion direction training and the white-noise stimulation but not the bottom-up

8 stimulation. (d) A summary of learning and transfer performance in training plus bottom-up

9 stimulation, bottom-up stimulation alone, and training plus noise-only conditions. (e) A

10 summary of the transfer indices in the baseline (replotted from Figure 2c), training plus

11 bottom-up stimulation, and training plus noise-only conditions. Error bars indicate \pm 1

12 standard errors of the means. ${ }^{* *} p<.01 ;{ }^{* *} p<.001$.

13 The first control experiment (Experiment 2B) indicated that the bottom-up stimulation

14 alone could not account for the performance improvement in the untrained orthogonal

15 motion direction. In this experiment, six new observers received an equal amount of

16 bottom-up stimulation in the orthogonal transfer motion direction without receiving training

17 with the motion discrimination task. The bottom-up stimulation did not significantly

18 decrease the motion direction discrimination threshold in the stimulated motion direction

19 (improved by $4.4 \pm 3.6 \%, t_{5}=1.24, p=.27,95 \% \mathrm{CI}=-4.7 \%$ to $13.6 \% ; p=.25$ with a

20 Wilcoxon test; Cohen's $d=.51$, Figures $3 \mathrm{~b}$ and $3 \mathrm{~d}$ ). 
The second control experiment (Experiment 2C) ruled out the possibility that the

2 dynamic white noise, which could have activated visual neurons that were tuned to all

3 motion directions, was sufficient to cause the same amount of learning transfer. The

4 experimental design was the same as that of Experiment $2 \mathrm{~A}$, except that no orthogonal

5 motion stimulus was present in the actual stimuli. Again, training reduced motion direction

6 discrimination threshold significantly by $44.0 \pm 3.0 \%\left(t_{5}=14.52, p<.001,95 \% \mathrm{CI}=36.2 \%\right.$

7 to $51.8 \% ; p=.028$ with a Wilcoxon test; Cohen's $d=5.93$ ) (Figures 3c and 3d). However,

8 learning transfer was not significant in the transfer motion direction $\left(10.0 \pm 7.8 \%, t_{5}=1.28\right.$,

$9 p=.26,95 \% \mathrm{CI}=-10.1 \%$ to $30.1 \% ; p=.25$ with a Wilcoxon test; Cohen's $d=.52$ ). This

10 transfer measure was also significantly lower than the improvement in the trained motion

11 direction $\left(t_{5}=6.59, p=.001,95 \% \mathrm{CI}=20.7 \%\right.$ to $47.3 \% ; p=.028$ with a Wilcoxon test;

12 Cohen's $d=2.69$ ). The TI was $0.17 \pm 0.18$ in this condition (Figure $3 \mathrm{e}$ ).

13 A Kruskal-Wallis test revealed significant differences among the TIs in the baseline

14 condition (Figure 2a), the training plus bottom-up stimulation condition (Figure 3a), and the

15 training plus noise-only condition (Figure 3c) $(p=.003)$. Post-hoc Dunn's multiple

16 comparisons indicated that the training plus bottom-up stimulation condition had

17 significantly more transfer than the baseline condition $(p=.015$, corrected in this and later

18 Post-hoc Dunn's multiple comparisons) and the training plus noise-only condition $(p=.007)$.

19 There was no significant difference between the TIs of the baseline and the 20 training-plus-noise-only conditions $(p=1.00)$. 

direction enabled significantly more transfer of motion direction learning than in the

3 baseline condition (Experiment 1), although transfer was incomplete. These results are

4 consistent with Zhang et al.'s study (2014), which suggested that motion direction learning

5 is likely to be a high-level process and could transfer to another direction as a result of

6 functional connectivity between high-level brain areas and sensory neurons tuned to the

7 transfer motion direction. Moreover, results in the current experiment further suggested that

8 the functional connectivity can be achieved via exposure to subliminal motion stimuli.

Experiment 3: Learning transfer - Effect of top-down attention

11 Top-down attention is often necessary for obtaining training-related performance

12 improvement (Ahissar \& Hochstein, 1993; Fahle, 1997, 2004). For example, when there

13 were two Vernier stimuli, discrimination accuracy only improved for the attended stimulus

14 but not the unattended stimulus (Fahle, 2004). In Experiment 3, we examined the effect of

15 the top-down attention on the learning transfer instead of the learning itself. Specifically, we

16 investigated whether learning in one motion direction, with the help of the top-down

17 attention to another motion direction (training plus top-down attention), would transfer to

18 the transfer motion direction. The results are summarized in Figure 4. Training and the

19 top-down attention to the transfer motion direction reduced motion direction threshold in the

20 trained motion direction by $39.3 \pm 3.2 \%\left(t_{5}=12.13, p<.001,95 \% \mathrm{CI}=31.0 \%\right.$ to $47.6 \% ; p$

$21=.028$ with a Wilcoxon test; Cohen's $d=4.95$, Experiment $3 \mathrm{~A}$ ) (Figures $4 \mathrm{a}$ and $4 \mathrm{c}$ ). The 
1 threshold reduction in the direction orthogonal to the training direction was also significant

$2 \quad\left(30.2 \pm 3.8 \%, t_{5}=8.04, p<.001,95 \% \mathrm{CI}=20.5 \%\right.$ to $39.9 \% ; p=.028$ with a Wilcoxon test;

3 Cohen's $d=3.28$ ), suggesting a learning transfer. Analysis based on null-hypothesis

4 significance test found that the improvement in the untrained motion direction was not

5 statistically different from that in the trained motion direction $\left(t_{5}=1.77, p=.137,95 \% \mathrm{CI}=\right.$

$6-4.1 \%$ to $22.3 \%$, Cohen's $d=.72$ ), with a TI value of $0.80 \pm 0.14$. This suggests a possibility

7 that the learning transfer was complete. However, a further Bayesian $t$-test found no

8 evidence for the hypothesis that there was any difference between the improvements in the

9 trained and untrained motion directions, $\mathrm{BF}_{10}=1.02$ (Marsman \& Wagenmakers, 2017),

10 with a Cauchy prior width of 0.707 in this (and also later) Bayesian $t$-tests. 
a

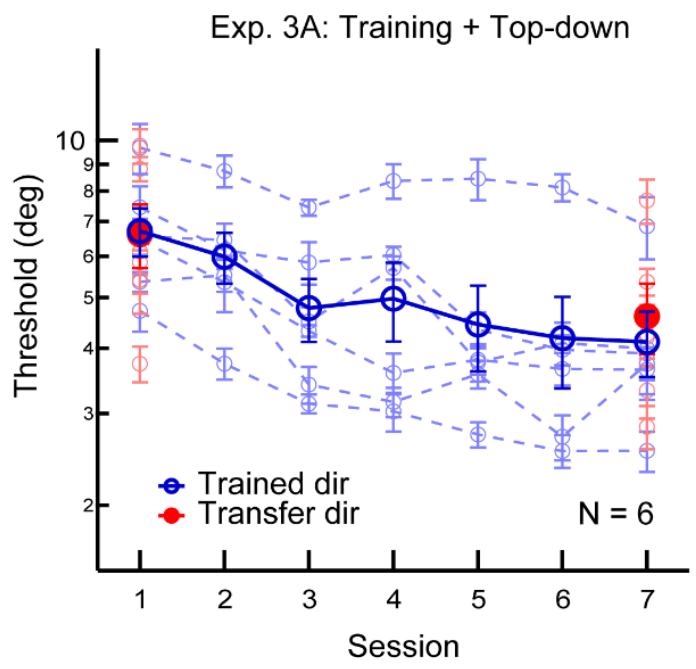

C

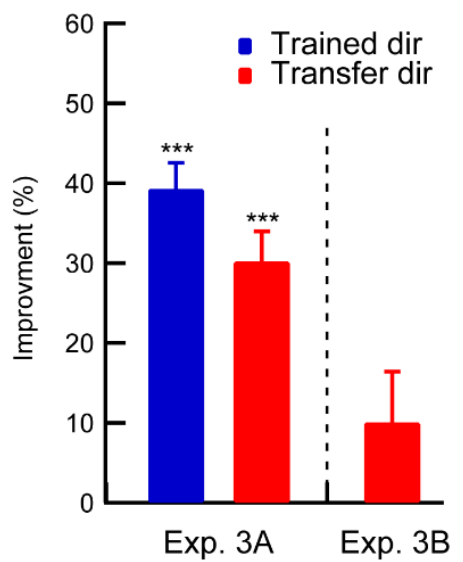

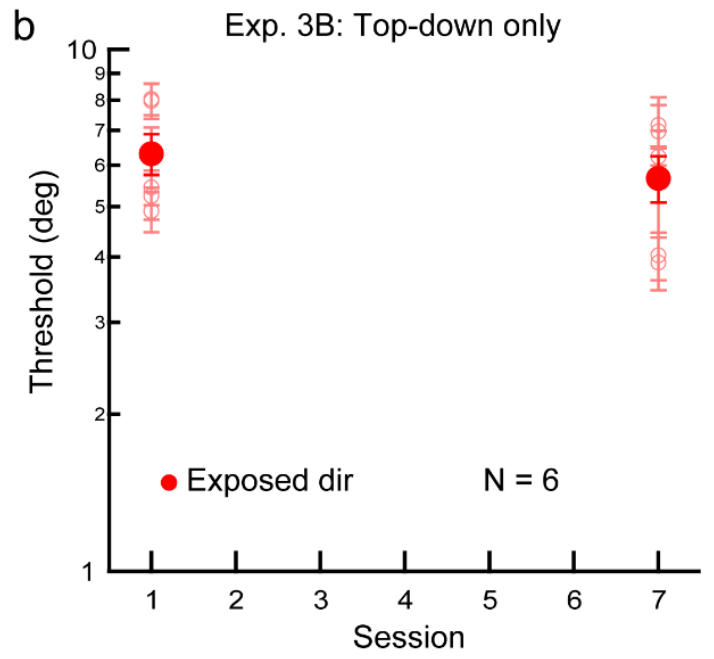

d

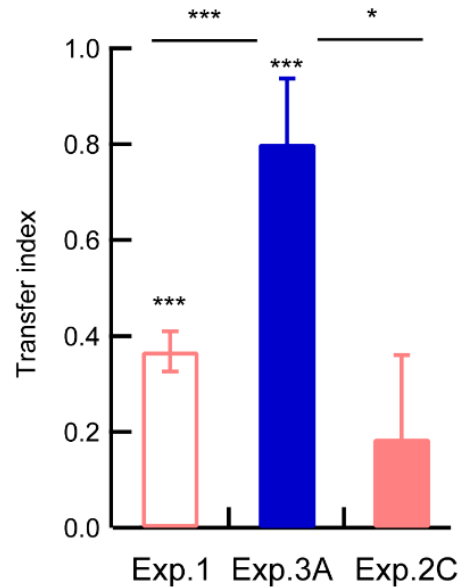

2 Figure 4. Results of Experiment 3, demonstrating the motion direction learning and the effect

3 of simultaneous top-down attention to the transfer motion direction. (a) The mean (solid line)

4 and individual (dash lines) learning and transfer data with training and the top-down

5 attention to the transfer motion direction (Experiment $3 \mathrm{~A}$ ). (b) Results of the control

6 experiment (Experiment 3B) without the motion discrimination training. (c) A summary of the

7 learning and transfer effects in the top-down attention and control experiments. (d) A

8 summary of the transfer indices in the training plus top-down attention condition, the

9 previous baseline condition (replotted from Figure $2 \mathrm{c}$ ), and the training plus noise-only condition (replotted from Figure $3 \mathrm{e}$ ). Error bars indicate \pm 1 standard errors of the means. ${ }^{*} p$ 
$1<.05 ;{ }^{* * *} p<.001$.

2 The control experiment (Experiment 3B) with six observers demonstrated that, without

3 actual training of motion direction discrimination, top-down attention to the transfer motion

4 direction alone could not lead to a significant learning effect in the transfer motion direction

$5 \quad\left(10.0 \pm 6.3 \%, t_{5}=1.59, p=.17,95 \% \mathrm{CI}=-6.2 \%\right.$ to $26.4 \% ; p=.17$ with a Wilcoxon test;

6 Cohen's $d=.65$ ) (Figures $4 \mathrm{~b}$ and $4 \mathrm{c}$ ).

7 A Kruskal-Wallis test revealed significant differences among the TIs in the baseline

8 condition (Figure 2a), the training plus top-down attention condition (Figure 4a), and the

9 training plus noise-only condition (Figure 3c) $(p=.008)$. Post-hoc Dunn's multiple

10 comparisons indicated that the training plus top-down attention condition had significantly

11 more transfer than the baseline condition $(p=.024)$ and the training plus noise-only

12 condition $(p=.021)$. The difference between the latter two conditions, however, did not

13 reach significance $(p=1.00)$.

14 These results jointly suggested that the top-down attention to the transfer motion

15 direction enabled significantly more transfer of the motion direction learning than transfer in

16 the baseline condition (Experiment 1). These results are consistent with the rule-based

17 learning model (Zhang et al., 2010), the notion that motion direction learning may be a

18 high-level process. The current finding further suggests that solely attending to the

19 anticipated motion signal (without any physical motion signal stimulating the visual cortex)

20 could functionally link the high-level perceptual learning and low-level neurons which turn

21 to the transfer direction. 
2 Experiment 4: Learning transfer - Effect of subsequent bottom-up stimulation

3 To explore whether the transfer-facilitation effect of the bottom-up stimulation and

4 top-down attention procedures on training could still be effective after learning have been

5 consolidated, we examined learning transfer by applying the motion discrimination training

6 and the bottom-up stimulation sequentially instead of in the same day.

7 The results are illustrated in Figure 5. Six new observers received training in the 8 training motion direction, and showed a significant training effect $\left(44.6 \pm 3.9 \%, t_{5}=11.3, p\right.$

$9<.001,95 \% \mathrm{CI}=34.5 \%$ to $54.8 \% ; p=.028$ with a Wilcoxon test; Cohen's $d=4.62$ )

10 (Figures 5a and 5b). Before the bottom-up stimulation, the learning significantly transferred

11 to the transfer motion direction $\left(21.4 \pm 2.6 \%, t_{5}=8.2, p<.001,95 \% \mathrm{CI}=14.7 \%\right.$ to $28.1 \%$;

$12 p=.028$ with a Wilcoxon test; Cohen's $d=3.33$ ), but significantly less than in the training

13 in the trained motion direction $\left(t_{5}=6.24, p=.002,95 \% \mathrm{CI}=13.7 \%\right.$ to $32.8 \% ; p=.028$ with

14 a Wilcoxon test; Cohen's $d=2.55$ ). This performance improvement in the transfer motion

15 direction may be caused by procedure learning and learning during the pre-test. After the

16 following bottom-up stimulation, the motion discrimination performance improved further

17 by $19.7 \pm 3.2 \%\left(t_{5}=6.20, p=.002,95 \% \mathrm{CI}=11.6 \%\right.$ to $27.9 \% ; p=.028$ with a Wilcoxon

18 test; Cohen's $d=2.53$ ) in the transfer motion direction. The overall improvement of the

19 transfer motion direction discrimination after training and subconscious bottom-up

20 stimulation was $36.9 \pm 3.2 \%\left(t_{5}=11.5, p<.001,95 \% \mathrm{CI}=28.7 \%\right.$ to $45.2 \% ; p=.028$ with a

21 Wilcoxon test; Cohen's $d=4.70)$ which was not different $\left(t_{5}=.78, p=.47,95 \% \mathrm{CI}=-9.0 \%\right.$ 
1 to $16.8 \%$, Cohen's $d=.32)$ from the training effect in the trained motion direction $(40.9 \pm$

$27.5 \%, t_{5}=5.45, p=.003,95 \% \mathrm{CI}=21.6 \%$ to $60.0 \% ; p=.028$ with a Wilcoxon test; Cohen's

$3 d=2.22$ ). Although the learning transferred largely to the transfer motion direction, the

4 Bayesian $t$-test showed no evidence for either supporting or rejecting the hypothesis that the

5 improvement differed between the trained and transfer motion directions $\left(\mathrm{BF}_{10}=.48\right)$.

6 The TI was $0.90 \pm 0.08$ (Figure $5 \mathrm{c}$ ), which was not statistically different $\left(t_{10}=.75, p\right.$

$7=.47,95 \% \mathrm{CI}=-.26$ to .13 , Cohen's $d=.44$ ) from that in the simultaneous bottom-up

8 stimulation condition (Experiment 2) $(\mathrm{TI}=0.83 \pm 0.03$, the blue bar in Figure $3 \mathrm{e})$. However,

9 a further Bayesian $t$-test did not show enough evidence for the two TIs being either the same

10 or different $\left(\mathrm{BF}_{10}=.56\right)$. The high level of learning transfer in the subsequent bottom-up

11 stimulation condition again suggests that the motion learning was more likely a high-level

12 process. More importantly, the result in this experiment demonstrates that the bottom-up

13 stimulation could also enable learning transfer after motion learning have been consolidated.

14

a

15

16

17

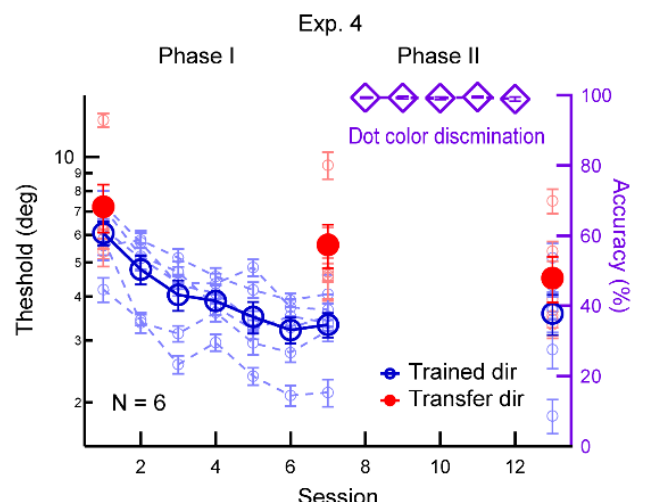

b

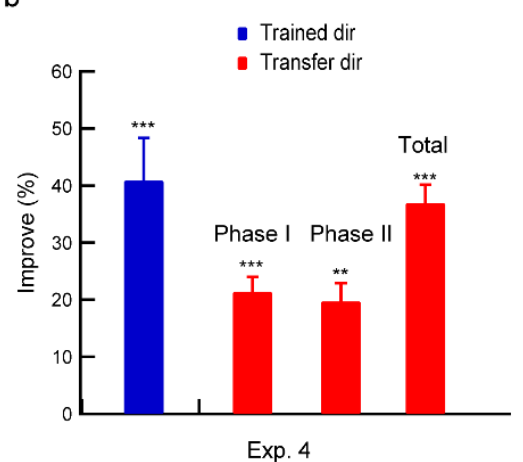

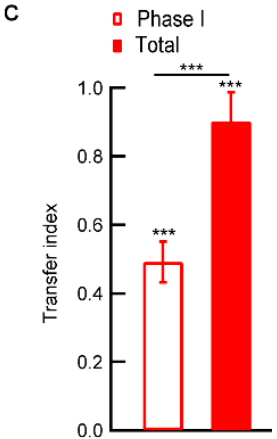

Exp. 4

Figure 5. Results of Experiment 4, showing the motion perceptual learning and the effect of subsequent bottom-up stimulation in the transfer motion direction. (a) The mean (solid line) and individual (dash lines) learning and transfer data with training and the subsequent 
1 top-down attention to the transfer motion direction. (b) A summary of the learning and

2 transfer effects. (c) A summary of the transfer indices. Error bars indicate \pm 1 standard errors

3 of the means. DE: dominant eye. ${ }^{* *} p<.01 ;{ }^{* *} p<.001$.

4 Experiment 5: Learning transfer - Effect of subsequent top-down attention

5 We also examined whether the sequential application of the motion discrimination

6 training and the top-down attention (to the transfer motion direction) could lead to a learning

7 transfer to the transfer motion direction. The results are demonstrated in Figure 6. The

8 observers were trained with the motion direction discrimination task for 5 sessions, and

9 showed a significant improvement $\left(51.5 \pm 2.6 \%, t_{6}=18.8, p<.001,95 \% \mathrm{CI}=44.8 \%\right.$ to

$1058.2 \% ; p=.018$ with a Wilcoxon test; Cohen's $d=7.11)$ in the trained motion direction. A

11 significant improvement in the untrained motion direction was also found $\left(16.6 \pm 4.6 \%, t_{6}=\right.$

$123.60, p=.011,95 \% \mathrm{CI}=5.3 \%$ to $27.9 \% ; p=.018$ with a Wilcoxon test; Cohen's $d=1.36$ ),

13 but significantly less than that in the trained motion direction $\left(t_{6}=9.38, p<.001,95 \% \mathrm{CI}=\right.$

$1425.8 \%$ to $43.9 \% ; p=.018$ with a Wilcoxon test; Cohen's $d=3.55$ ). The transfer motion

15 direction was subsequently attended to in a motion detection task. This led to a further

16 performance improvement of $19.8 \% \pm 2.9 \%\left(t_{6}=6.50, p=.001,95 \% \mathrm{CI}=12.4 \%\right.$ to $27.3 \%$;

$17 p=.018$ with a Wilcoxon test; Cohen's $d=2.46$ ). The overall improvement in the transfer

18 motion direction after training and the top-down attention sessions was $33.9 \pm 1.9 \%\left(t_{6}=\right.$

$1916.5, p<.001,95 \% \mathrm{CI}=28.9 \%$ to $38.8 \% ; p=.018$ with a Wilcoxon test; Cohen's $d=6.25$ ),

20 significantly less $\left(t_{6}=5.16, p=.002,95 \% \mathrm{CI}=6.9 \%\right.$ to $19.3 \% ; p=.018$ with a Wilcoxon

21 test; Cohen's $d=1.95)$ than that in the trained motion direction $\left(46.9 \pm 3.4 \%, t_{6}=13.9, p\right.$ 
$1<.001,95 \% \mathrm{CI}=38.7 \%$ to $55.2 \% ; p=.018$ with a Wilcoxon test; Cohen's $d=5.27$,

2 indicating a substantial but incomplete learning transfer.

3 The TI was $0.73 \pm 0.04$ (Figure $6 \mathrm{c}$ ), which was not different $\left(t_{11}=.53, p=.61,95 \% \mathrm{CI}\right.$

$4=-.22$ to .36 , Cohen's $d=.29$ ) from that in the simultaneous top-down attention condition

$5 \quad(\mathrm{TI}=0.80 \pm 0.14$, the blue bar in Figure $4 \mathrm{~d})$. However, a further Bayesian $t$-test did not

6 show enough evidence for the two TIs being either the same or different $\left(\mathrm{BF}_{10}=.50\right)$. These

7 results convergingly suggest that the top-down attention manipulation could also enable

8 learning transfer after motion learning have been consolidated.

a

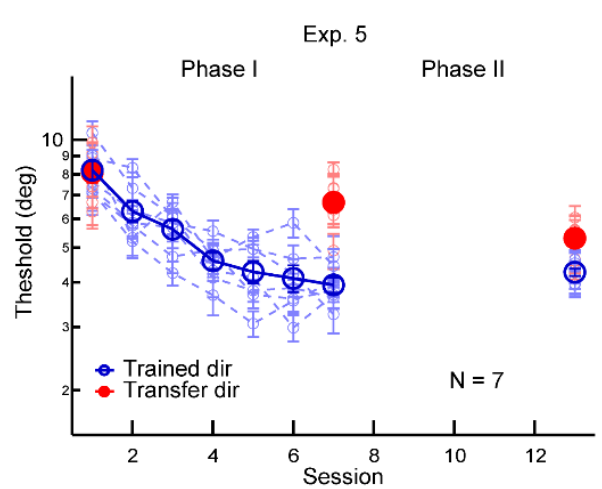

b

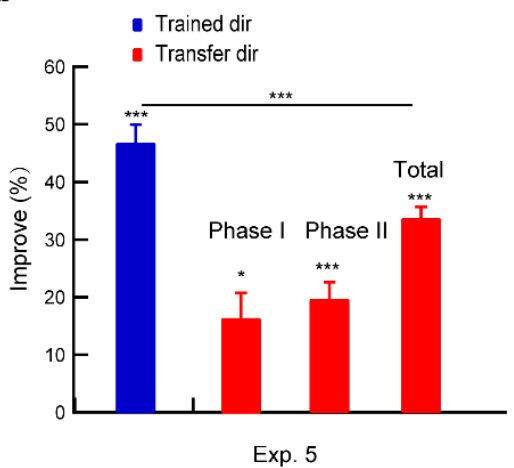

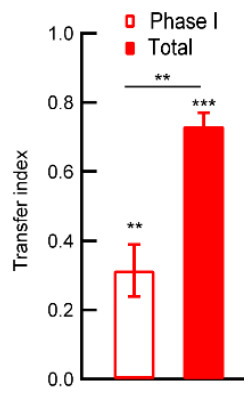

Exp. 5

10 Figure 6. Results of Experiment 5 about the learning and transfer effects of the motion

11 direction discrimination training and the subsequent top-down attention to the transfer

12 motion direction. (a) The mean (solid line) and individual (dash lines) learning and transfer

13 data with training and subsequent top-down attention to the transfer motion direction. (b) A

14 summary of the learning and transfer effects. (c) A summary of the transfer indices. Error

15 bars indicate \pm 1 standard errors of the means. DE: dominant eye. ${ }^{*} p<.05 ;{ }^{* *} p<.01 ;{ }^{* * *} p$ $16<.001$.

\section{Discussion}


It has been previously found that bottom-up stimulation and top-down attention

2 facilitated learning transfer of form perception (Xiong, Zhang, et al., 2016). The current

3 study extended our understanding by showing that transfer of motion perception could also

4 occur following the bottom-up stimulation and top-down attention manipulations. More

5 importantly, learning transfer observed in the current study suggested that bottom-up

6 stimulation and top-down attention could still be effective after learning have already been

7 consolidated, because the transfer effect was significant even when training and the

8 bottom-up stimulation/top-down attention were conducted sequentially across days. These

9 results offered new insight into the rule-based model (Zhang et al., 2010) by suggesting that

10 functional connectivity between the high level areas and low-level areas which response to

11 transfer motion direction could be established after learning have already been consolidated.

12 The current results are consistent with Xiong, Zhang et al.'s (2016) study in that

13 learning can transfer to untrained conditions when training is accompanied by passive

14 bottom-up stimulation of the transfer conditions. However, the results are at odds with

15 previous studies by Mastropasqua et al. (2015) and Wang et al. (2012), who reported that

16 passive stimulation in the transfer condition was insufficient to elicit a learning transfer. The

17 most likely explanation for this discrepancy is that the CFS used in the current study and

18 Xiong, Zhang et al.'s (2016) study rendered the passively exposed stimuli subconscious.

19 This could potentially make the stimuli escape suppression from the attention system, or at

20 least make the involvement of the attention system greatly reduced. In contrast, the passively

21 exposed stimuli (as in Mastropasqua et al., 2015) were above the detection threshold, thus 
1 could be actively inhibited by attention (Watanabe, Nanez, \& Sasaki, 2001). Unfortunately,

2 this explanation fails to explain why only limited learning transfer was found in a more

3 recent study (Lange \& De Weerd, 2018) wherein the untrained location was also

4 unconsciously exposed. A careful comparison showed that there are at least two crucial

5 differences between Lange and De Weerd's (2018) study and Xiong, Zhang et al.'s (2016)

6 study. Firstly, Lange and De Weerd used more training sessions (15 sessions) than Xiong,

7 Zhang et al. (6 sessions). Previous studies (Jeter, Dosher, Liu, \& Lu, 2010) demonstrated

8 that learning specificity could be enhanced by extensive training. Therefore, the differential

9 transfer effects between these two studies may be accounted for by different amounts of

10 training. Secondly, these two studies quantified learning transfer in different ways. Lange

11 and De Weerd (2018) considered significant improvement in the transfer condition after

12 post-test as an index of specificity, whereas Xiong, Zhang et al. (2016) used the relative

13 improvement between the transfer condition and the trained condition as an index of

14 specificity. The discrepancy in their choices of the definition of specificity (and accordingly

15 the analysis methods) may have also contributed to the diverging conclusions.

16 Perceptual learning may even occur when people were trained (or were only required to

17 imagine) to discriminate between identical stimuli (Amitay, Irwin, \& Moore, 2006;

18 Grzeczkowski, Tartaglia, Mast, \& Herzog, 2015). However, the visual learning transfer was

19 limited if there was not enough variance in the feature of the stimuli (e.g., motion direction),

20 even if the double-training paradigm was used (Liang, Zhou, Fahle, \& Liu, 2015a).

21 Observers might use some subtle local cues (e.g., edges of a monitor) during training with 
1 limited variance in the stimuli (Zhang, Xiao, Klein, Levi, \& Yu, 2010). When a feature of the

2 stimuli (e.g., motion direction) had some jitter (as in a staircase procedure), there was

3 significantly more learning transfer than when there was little feature variance (Xiong, Xie

4 et al., 2016). Consistent with Xiong, Xie et al.'s (2016) study, Green, Kattner, Siegel,

5 Kersten, and Schrater (2015) found that a relatively less varied simple

6 orientation-categorization training task resulted in orientation-specific learning, whereas a

7 more varied orientation-estimation task showed orientation-general learning. Another study

8 (Liang, Zhou, Fahle, \& Liu, 2015b) found that even with a staircase method, transfer of

9 motion learning under double-training was not greater than the control condition. However,

10 a meta-analysis (Zhang \& Yu, 2016) suggested that the results in Liang et al.'s (2015b) study

11 was not statistically different from those of Zhang et al. (2014) and Xiong, Xie, et al.'s (2016)

12 studies. The meta-analysis exhibited an overall TI of 0.78 , comparable to the TIs found in

13 the current study (TI $=0.83$ in the double-training condition of Experiment 2, the blue bar in

14 Figure 3e; TI $=0.80$ in Experiment 3, the blue bar in Figure 4d; TI $=0.90$ in Experiment 4,

15 the solid bar in Figure 5c, and TI $=0.73$ in Experiment 5, the solid bar in Figure 6c).

16 Although motion perceptual learning has been studied for decades, the controversy

17 about the associated brain areas remains. Nishina et al. (2009) suggested that motion

18 perceptual learning occurs in the primary visual cortex in which neurons are particularly

19 sensitive to local motion signals. Besides, the area of V3A has also been suggested to be

20 involved in global motion detection learning (Shibata et al., 2012). On the other hand,

21 electrophysiological studies (Britten, Shadlen, Newsome, \& Movshon, 1992; Newsome, 
1 Britten, Salzman, \& Movshon, 1990) demonstrated that the middle temporal (MT) area in

2 monkeys is particularly sensitive to the motion direction of global motion patterns. This was

3 further confirmed by other studies showing that lesions in the MT and medial superior

4 temporal (MST) areas in monkeys (Newsome \& Pare, 1988) and humans (Vaina, Lemay,

5 Bienfang, Choi, \& Nakayama, 1990) impaired perception of global motion direction. It

6 could be hypothesized that MT/MST (often referred to as MT+ in humans) are responsible

7 for motion direction discrimination learning. This notion is also supported by

8 psychophysical (Salzman, Britten, \& Newsome, 1990) and fMRI studies (Zohary, Celebrini,

9 Britten, \& Newsome, 1994). However, it is important to note that motion learning activating

10 some brain areas does not necessarily mean that motion learning takes place in these areas.

11 On the contrary, motion learning can also occur in higher brain areas which send feedback

12 information to these lower visual areas (Mollon \& Danilova, 1996). This hypothesis is

13 supported by a neuroimaging study (Law \& Gold, 2008) in which motion perceptual

14 learning was accompanied by brain activities in lateral intraparietal (LIP) neurons instead of

15 MT+.

16 Consistent with Law and Gold's (2008) study, Zhang and Yang (2014) found that

17 motion discrimination learning transferred significantly (sometimes completely) to the

18 direction opposite to the training direction if participants were passively exposed to the

19 opposite direction. It was suggested that the motion discrimination learning might be a

20 high-level process and that the learning was potentially transferrable rules of re-weighting

21 the motion direction inputs (Zhang et al., 2010). The authors further speculated that brain 
1 areas involved in high-level learning are not always functionally connected to the sensory

2 neurons sensitive to other directions, thus leading to direction learning specificity; when

3 there is exposure to motion stimuli in an untrained direction, the functional connectivity

4 with neurons sensitive to the untrained motion direction can be fostered. The top-down

5 connections between high-level learning and low-level visual neurons can be reflected by

6 the ERP P1-N1 changes (Zhang, Cong, Song, \& Yu, 2013). Our current findings further

7 suggest that even subconscious stimulation or top-down attention in an untrained motion

8 direction is sufficient for establishing the task specific functional connectivity.

9 A series of perceptual learning studies with task-irrelevant features had shown that

10 learning only occurred when the irrelevant features were temporally paired with rewards in

11 the trained tasks (Seitz \& Watanabe, 2003; Watanabe et al., 2001). The stimulus-reward

12 pairing is suggested to be the mechanism of the learning of task-irrelevant features (Seitz \&

13 Watanabe, 2005). However, as discussed earlier, if the untrained and the trained features

14 (e.g., different motion directions) are displayed simultaneously, to enable learning transfer to

15 the untrained feature, the untrained feature should be below the discrimination threshold or

16 awareness so that it will not be suppressed by attention. If the untrained feature is

17 suprathreshold, to avoid attentional suppression, the stimuli carrying the untrained feature

18 should not be presented simultaneously with the trained stimuli (Zhang et al., 2010). The

19 current study provided further evidence that training plus subsequent sub-threshold exposure

20 of untrained features can lead to successful learning transfer.

21 Last but not least, the top-down attention to the untrained feature had a similar effect. It 
1 is worth pointing out that when the bottom-up stimulation/top-down attention and training

2 were separated by days, training preceding the bottom-up stimulation or top-down attention

3 transferred to the untrained feature. However, it is still unclear whether the same effect will

4 be found with a reversed task order (i.e., the bottom-up stimulation/top-down attention

5 before training). According to a previous study (Zhang et al., 2010), this is unlikely because

6 the function of the bottom-up stimulation and the top-down attention is theorized only to

7 consolidate transfer of the learning effect, which should have been achieved beforehand.

\section{Acknowledgment}

9 This research was supported by the National Natural Science Foundation of China 10 (31600872 and 31300833), the MOE (Ministry of Education in China) Project of Humanities 11 and Social Sciences (19YJC190030), the Social Science Foundation of Jiangsu Province 12 (17JYC006), and the City \& University strategy-Soochow University Leading Research Team 13 in Humanities and Social Sciences.

\section{References}

15 Ahissar M, Hochstein S. (1993) Attentional control of early perceptual learning. 16 Proceedings of the National Academy of Sciences. 90(12), 5718-22.

17 Ahissar, M., \& Hochstein, S. (1997). Task difficulty and the specificity of perceptual 18 learning. Nature, 387(6631), 401-406.

19 Amitay, S., Irwin, A., \& Moore, D. R. (2006). Discrimination learning induced by training 20 with identical stimuli. Nature Neuroscience, 9(11), 1446-1448.

21 Ball, K., \& Sekuler, R. (1987). Direction-specific improvement in motion discrimination. Vision Research, 27(6), 953-965.

Brainard, D. H. (1997). The Psychophysics Toolbox. Spatial Vision, 10(4), 433-436.

Britten, K. H., Shadlen, M. N., Newsome, W. T., \& Movshon, J. A. (1992). The analysis of visual motion: a comparison of neuronal and psychophysical performance. Journal of Neuroscience, 12(12), 4745-4765.

Fahle, M. (1994). Human pattern recognition: parallel processing and perceptual learning. Perception, 23(4), 411-427.

29 Fahle, M. (1997). Specificity of learning curvature, orientation, and vernier discriminations. 
Vision Research, 37(14), 1885-1895.

Fahle, M. (2004). Perceptual learning: A case for early selection. Journal of Vision, 4(10), 879-890.

Fahle, M., \& Poggio, T. A. (2002). Perceptual Learning. Cambridge: MIT Press.

Fiorentini, A., \& Berardi, N. (1981). Learning in grating waveform discrimination: specificity for orientation and spatial frequency. Vision Research, 21(7), 1149-1158.

Ghose, G. M., Yang, T., \& Maunsell, J. H. (2002). Physiological correlates of perceptual learning in monkey V1 and V2. Journal of Neurophysiology, 87(4), 1867-1888.

Gilbert, C. D., Sigman, M., \& Crist, R. E. (2001). The neural basis of perceptual learning. Neuron, 31(5), 681-697.

Green, C. S., Kattner, F., Siegel, M. H., Kersten, D., \& Schrater, P. R. (2015). Differences in perceptual learning transfer as a function of training task. Journal of Vision, 15(10), 5.

Herzog, M. H., Cretenoud, A. F., \& Grzeczkowski, L. (2017). What is new in perceptual learning? Journal of Vision, 17(1), 23.

Hubel, D. H., \& Wiesel, T. N. (1959). Receptive fields of single neurones in the cat's striate cortex. The Journal of Physiology, 148, 574-591.

Jeter, P. E., Dosher, B. A., Liu, S. H., \& Lu, Z. L. (2010). Specificity of perceptual learning increases with increased training. Vision Research, 50(19), 1928-40

Karni, A., \& Sagi, D. (1991). Where practice makes perfect in texture discrimination: evidence for primary visual cortex plasticity. Proceedings of the National Academy of Sciences, 88(11), 4966-4970.

Lange, G., \& De Weerd, P. (2018). Limited transfer of visual skill in orientation discrimination to locations treated by pre-testing and subliminal exposure. Vision Research, 143, 103-116.

Law, C. T., \& Gold, J. I. (2008). Neural correlates of perceptual learning in a sensory-motor, but not a sensory, cortical area. Nature Neuroscience, 11(4), 505-513.

Liang, J., Zhou, Y., Fahle, M., \& Liu, Z. (2015a). Limited transfer of long-term motion perceptual learning with double training. Journal of Vision, 15(10), 1.

Liang, J., Zhou, Y., Fahle, M., \& Liu, Z. (2015b). Specificity of motion discrimination learning even with double training and staircase. Journal of Vision, 15(10), 3.

Logothetis, N. K. (1994) Physiological studies of motion inputs. In: Visual detection of motion, ed. A. T. Smith. Academic Press. Marsman, M., \& Wagenmakers, E. (2017). Bayesian benefits with JASP. European Journal of Developmental Psychology, 14(5), 545-555.

Mastropasqua, T., Galliussi, J., Pascucci, D., \& Turatto, M. (2015). Location transfer of perceptual learning: passive stimulation and double training. Vision Research, 108, 93-102.

Mollon, J. D., \& Danilova, M. V. (1996). Three remarks on perceptual learning. Spatial Vision, 10(1), 51-58.

Newsome, W. T., Britten, K. H., Salzman, C. D., \& Movshon, J. A. (1990). Neuronal mechanisms of motion perception. Cold Spring Harbor Symposia on Quantitative 
Biology, 55, 697-705.

Newsome, W. T., \& Pare, E. B. (1988). A selective impairment of motion perception following lesions of the middle temporal visual area (MT). Journal of Neuroscience, $8(6), 2201-2211$.

Nishina, S., Kawato, M., \& Watanabe, T. (2009). Perceptual learning of global pattern motion occurs on the basis of local motion. Journal of Vision, 9(9), 15 11-16.

Peigneux, P., \& Smith, C. (2010). Memory processing in relation to sleep. In M. Kryger, T. Roth, \& W. Dement (Eds.), Principles and practice of sleep medicine (17th ed., pp. 335-347). Philadelphia: Elsevier.

Pelli, D. G. (1997). The VideoToolbox software for visual psychophysics: transforming numbers into movies. Spatial Vision, 10(4), 437-442.

Petrov, A. A., Dosher, B. A., \& Lu, Z. L. (2005). The dynamics of perceptual learning: an incremental reweighting model. Psychological Review, 112(4), 715-743.

Salzman, C. D., Britten, K. H., \& Newsome, W. T. (1990). Cortical microstimulation influences perceptual judgements of motion direction. Nature, 346(6280), 174-177.

Schoups, A., Vogels, R., \& Orban, G. (1995). Human perceptual learning in identifying the oblique orientation: retinotopy, orientation specificity and monocularity. The Journal of physiology, 483(3), 797-810.

Schoups, A., Vogels, R., Qian, N., \& Orban, G. (2001). Practising orientation identification improves orientation coding in V1 neurons. Nature, 412(6846), 549-553.

Seitz, A. R., \& Watanabe, T. (2003). Psychophysics: Is subliminal learning really passive? Nature, 422(6927), 36.

Seitz, A. R., \& Watanabe, T. (2005). A unified model for perceptual learning. Trends in Cognitive Sciences, 9(7), 329-334.

Shibata, K., Chang, L. H., Kim, D., Nanez, J. E., Sr., Kamitani, Y., Watanabe, T., \& Sasaki, Y. (2012). Decoding reveals plasticity in V3A as a result of motion perceptual learning. PLoS One, 7(8), e44003.

Teich, A. F., \& Qian, N. (2003). Learning and adaptation in a recurrent model of V1 orientation selectivity. Journal of Neurophysiology, 89(4), 2086-2100.

Tootell, R. B., Silverman, M. S., Switkes, E., \& De Valois, R. L. (1982). Deoxyglucose analysis of retinotopic organization in primate striate cortex. Science, 218(4575), 902-904.

Tsuchiya, N., \& Koch, C. (2005). Continuous flash suppression reduces negative afterimages. Nature Neuroscience, 8(8), 1096-1101.

Vaina, L. M., Lemay, M., Bienfang, D. C., Choi, A. Y., \& Nakayama, K. (1990). Intact "biological motion" and "structure from motion" perception in a patient with impaired motion mechanisms: a case study. Visual Neuroscience, 5(4), 353-369.

Wang, R., Zhang, J. Y., Klein, S. A., Levi, D. M., \& Yu, C. (2012). Task relevancy and demand modulate double-training enabled transfer of perceptual learning. Vision Research, 61, 33-38.

Watanabe, T., Nanez, J. E., \& Sasaki, Y. (2001). Perceptual learning without perception. Nature, 413(6858), 844-848. 
Xiao, L. Q., Zhang, J. Y., Wang, R., Klein, S. A., Levi, D. M., \& Yu, C. (2008). Complete transfer of perceptual learning across retinal locations enabled by double training. Current Biology, 18(24), 1922-1926.

Xiong, Y. Z., Xie, X. Y., \& Yu, C. (2016). Location and direction specificity in motion direction learning associated with a single-level method of constant stimuli. Vision Research, 119, 9-15.

Xiong, Y. Z., Zhang, J. Y., \& Yu, C. (2016). Bottom-up and top-down influences at untrained conditions determine perceptual learning specificity and transfer. eLife, 5.

Zhang, G. L., Cong, L. J., Song, Y., \& Yu, C. (2013). ERP P1-N1 changes associated with Vernier perceptual learning and its location specificity and transfer. Journal of Vision, 13(4), 19.

Zhang, G.-L., Li, A.-S., Miao, C.-G., He, X., Zhang, M., \& Zhang, Y. (2018). A consumer-grade LCD monitor for precise visual stimulation. Behavior Research Methods, 50(4), 1496-1502.

Zhang, J.-Y., \& Yang, Y.-X. (2014). Perceptual learning of motion direction discrimination transfers to an opposite direction with TPE training. Vision Research, 99, 93-98.

Zhang, J.-Y., Zhang, G.-L., Xiao, L.-Q., Klein, S. A., Levi, D. M., \& Yu, C. (2010). Rule-based learning explains visual perceptual learning and its specificity and transfer. Journal of Neuroscience, 30(37), 12323-12328.

Zhang, J.-Y., \& Yu, C. (2016). The transfer of motion direction learning to an opposite direction enabled by double training: A reply to Liang et al. (2015). Journal of Vision, 16(3), 29.

Zhang, T., Xiao, L. Q., Klein, S. A., Levi, D. M., \& Yu, C. (2010). Decoupling location specificity from perceptual learning of orientation discrimination. Vision Research, 50(4), 368-374.

Zohary, E., Celebrini, S., Britten, K. H., \& Newsome, W. T. (1994). Neuronal plasticity that underlies improvement in perceptual performance. Science, 263(5151), 1289-1292. 